\title{
Internal Energy Level Population Redistribution of Carbon Dioxide in Laminar and Turbulent Flow
}

\author{
Wisely Wong, ${ }^{1}$ Zhiyu Yang, ${ }^{1}$ and Amador Muriel ${ }^{2,3}$ \\ ${ }^{1}$ Department of Physics, Hong Kong University for Science and Technology, Kowloon, Hong Kong \\ ${ }^{2}$ Department of Electrical Engineering, Columbia University, New York, NY, USA \\ ${ }^{3}$ Department of Philosophy, Harvard University, Cambridge, MA 02138, USA \\ Correspondence should be addressed to Wisely Wong; wisely@ust.hk
}

Received 19 February 2013; Accepted 21 March 2013

Academic Editors: A. Huczynski and M. Mączka

Copyright (C) 2013 Wisely Wong et al. This is an open access article distributed under the Creative Commons Attribution License, which permits unrestricted use, distribution, and reproduction in any medium, provided the original work is properly cited.

We show clear experimental evidence that in the laminar flow regime, there is a continuous redistribution of population on different vibrorotational energy levels as the flow rate increases. Such redistribution comes to an abrupt stop when the flow changes to turbulence. The population distribution then remains almost unchanged even up to the flow rate 10 times the laminar to turbulent transition. The flow status of carbon dioxide is therefore closely related to its internal energy level population distribution.

In the past ten years, several experiments have been performed to test the long-accepted idea that when expressed in terms of dimensionless variables, such as the critical Reynolds number, the laminar-turbulent transition is independent of the fluid used in the experiment, a principle sometimes referred to as scale invariance, a fundamental tenet enshrined in the study of turbulence [1]. The experiments are of two types: (I) quasistatic [2-7] and (II) unconstrained free efflux [8-10]. Type I experiments have mixed results: $[6,7]$ support scale invariance, while [2-5] raise doubts on its validity. Type II experiments, using modern apparatus from the field of vacuum science and technology, are supportive of the theoretical studies which propose a quantum origin for a theory of turbulence [11-13]. The results of Type II experiments support the assertion that the critical Reynolds number, at which the laminar-turbulent transition occurs, is specie dependent. Thus, normal and heavy water have different critical Reynolds numbers, and so do the noble gases.

Scale invariance and specie dependence are contrary to each other. Scale invariance comes from the classical continuum approach, proposed before atoms and molecules were discovered, while specie dependence is characteristic of a quantum view of nature. It is important to settle this controversy.
In this paper, we report a Type III experiment: simultaneous spectroscopic measurements that compare the absorption spectra of an excited gas and its state of motion, in laminar or turbulent form. We have designed an experiment that correlates different populations of $\mathrm{CO}_{2}$ gas. There is only one species, pure $\mathrm{CO}_{2}$, but it has subspecies, namely, the different ground and excited states of this molecule. The tests in this experiment are more stringent than Type I and Type II experiments and certainly go beyond a classical continuum model.

The experimental schematics are shown in Figure 1. The gas cell was made of stainless steel with inner dimensions of $10 \mathrm{~mm} \times 10 \mathrm{~mm} \times 500 \mathrm{~mm}$. The infrared windows were made of $\mathrm{KBr}$ by directly pressing the $\mathrm{KBr}$ powder into the steel wall openings of the gas cell. Their inner surfaces were leveled with the inner wall of the cell, which was mechanically polished, to prevent extra friction to gas flow. The overall transmission of the pair of windows was about $10 \%$. The windows were at $50 \mathrm{~mm}$ from the inlet of the gas cell. The inner diameter of the gas tubes connected to the inlet and the outlet of the cell was $4.5 \mathrm{~mm}$. The $\mathrm{CO}_{2}$ gas ( $99.5 \%$ purity) first passed through a flow meter (MKS 149A), then a pressure gauge (MKS 872B) before entering the gas cell. To get more accurate readings at low flow rates, a voltmeter (Keithley 2000) was used to measure the DC voltage output of the 


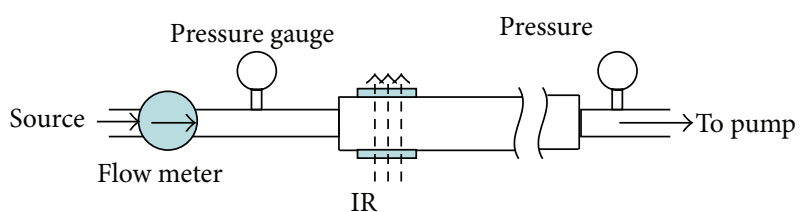

FIGURE 1: Schematics of the experimental setup.

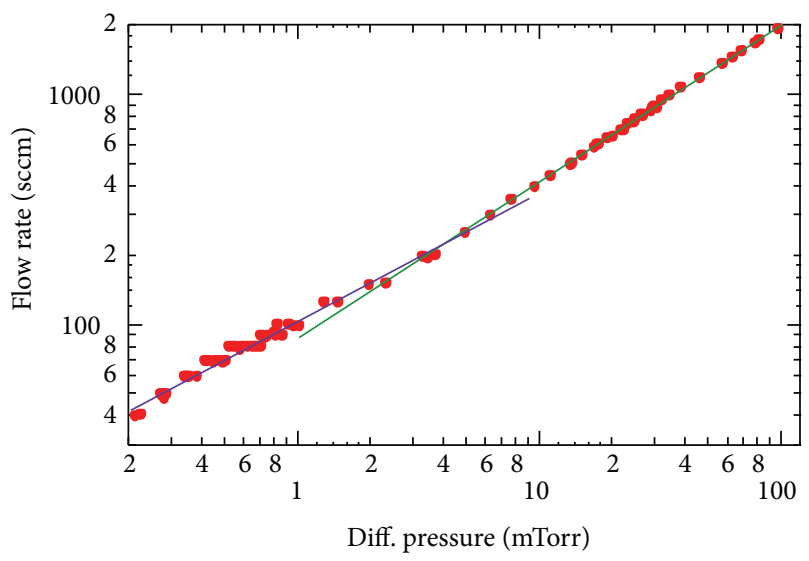

FIGURE 2: Measured flow rate as a function of pressure difference between the entrance and the exit of the gas cell in log-log plot. The straight lines are linear fits.

flow meter. Another pressure gauge (MKS 872B) measured the downstream pressure, while the outlet gas entered a buffer volume of about $3000 \mathrm{~cm}^{3}$ before being pumped to the atmosphere by a mechanical pump. The portion of the cell with the windows was placed in the sample chamber of a Fourier transform spectrometer (Bruker IFS-66) for transmission measurements in the 600 to $7000 \mathrm{~cm}^{-1}$ spectral range.

The flow rate versus the pressure difference between the inlet and the outlet is plotted in Figure 2. Two regimes of flow can be distinguished. In the low flow rate regime, a slope of 1.48 can be extracted from the log-log plot, and in the high flow rate regime, the slope is 1.78 . This is consistent with the general trend that in turbulence the flow rate is higher than that in laminar flow under the same pressure difference. The transition from laminar to turbulence occurs at around 200 standard cubic centimeters per minute ( $\mathrm{sccm}$ ), which agrees with the lower end of the simple theoretical estimation for an infinitely long gas cell of $10 \mathrm{~mm} \times 10 \mathrm{~mm}$ in cross-section. The discontinuity in cross-section from $4.5 \mathrm{~mm}$ circular diameter to $10 \mathrm{~mm} \times 10 \mathrm{~mm}$ square will cause the turbulence to appear at lower flow rates.

$\mathrm{CO}_{2}$ gas exhibits rich vibrational and rotational spectroscopic absorption lines in the infrared [14-19]. The changes in the relative intensity of these lines reflect the populations at different molecular energy levels. To detect the possible change of population distribution due to laminar to turbulent transition, it is, therefore, necessary to measure and analyze carefully the infrared spectra at different flow conditions. Typical transmission spectra of $\mathrm{CO}_{2}$ gas in the cell and that of

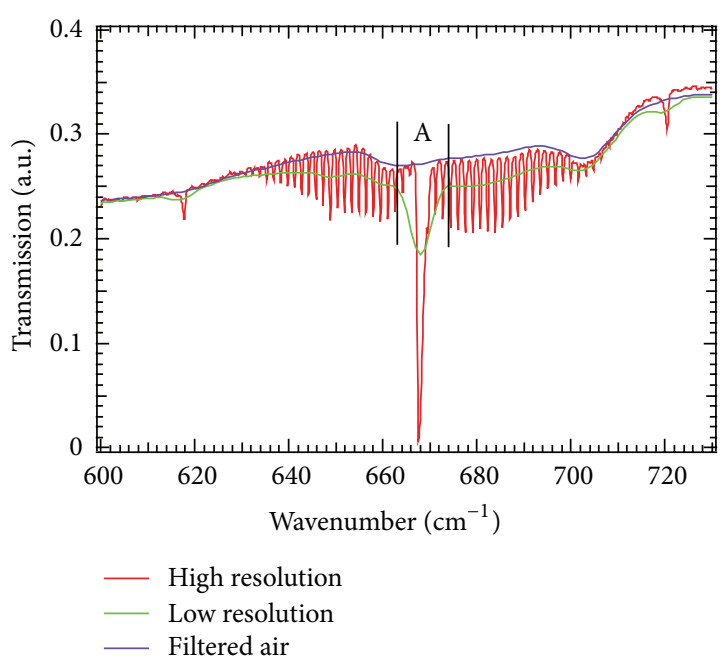

(a)

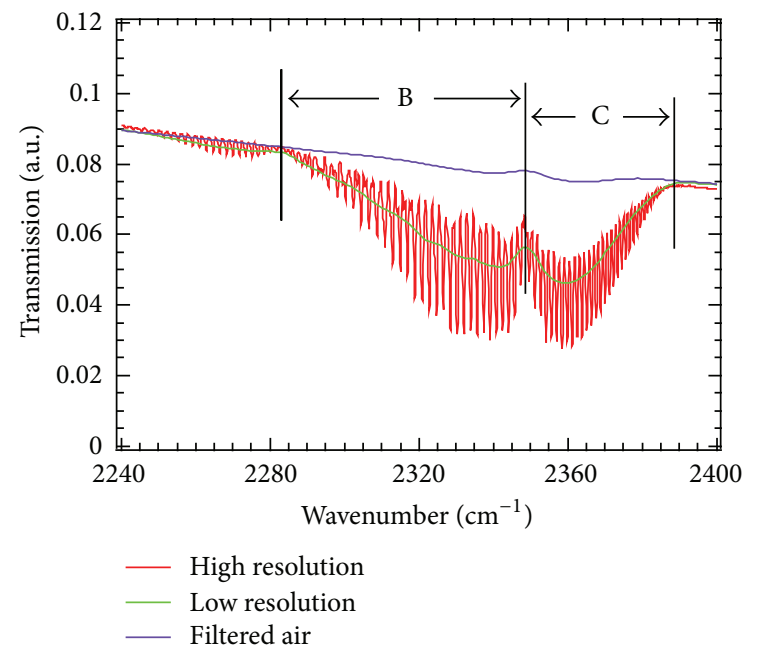

(b)

FIgURE 3: (a) The absorption spectra and the background spectrum around the first major absorption band of $\mathrm{CO}_{2}$ gas. (b) The absorption spectra and the background spectrum around the second and the third major absorption bands of $\mathrm{CO}_{2}$ gas.

background $\left(\mathrm{CO}_{2}\right.$ and water vapor depleted air) at different spectral resolutions are shown in Figure 3. Figure 3(a) shows the spectra around the first major $\mathrm{CO}_{2}$ absorption line near $668 \mathrm{~cm}^{-1}$, which is due to the transition from the ground state (00001) to the first vibrational excited state (01101) [14] along with the rotational side bands. In the high resolution $\left(0.1 \mathrm{~cm}^{-1}\right)$ spectrum, a number of weaker and narrow absorption lines involving the rotational energy levels can be identified. In the low resolution spectrum $\left(10 \mathrm{~cm}^{-1}\right)$, these weaker lines are smeared out, and the first major line becomes wider and shallower, while maintaining the total strength (area). The absorption $A$ is related to the transmission $T$ in the conventional way, that is, $A=-\log T$. We define the band-A absorption $I_{A}$ as the absorption between 663 and $647 \mathrm{~cm}^{-1}$ as indicated in Figure 3(a). The average pressure in the cell was 

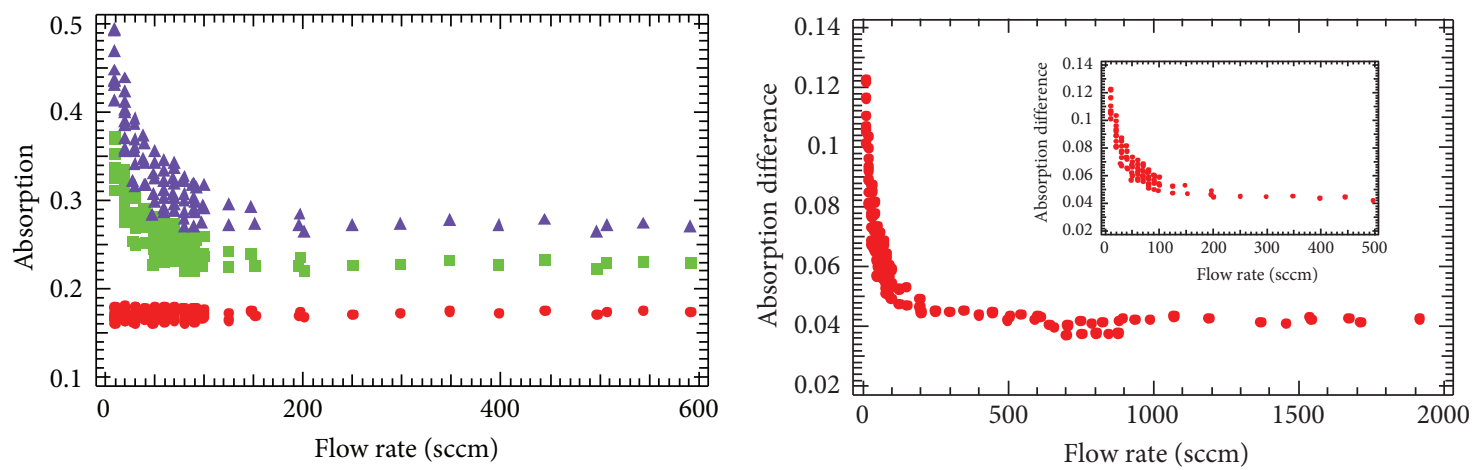

- Band-A

- Band-B

$\Delta$ Band-C

(a)

(b)

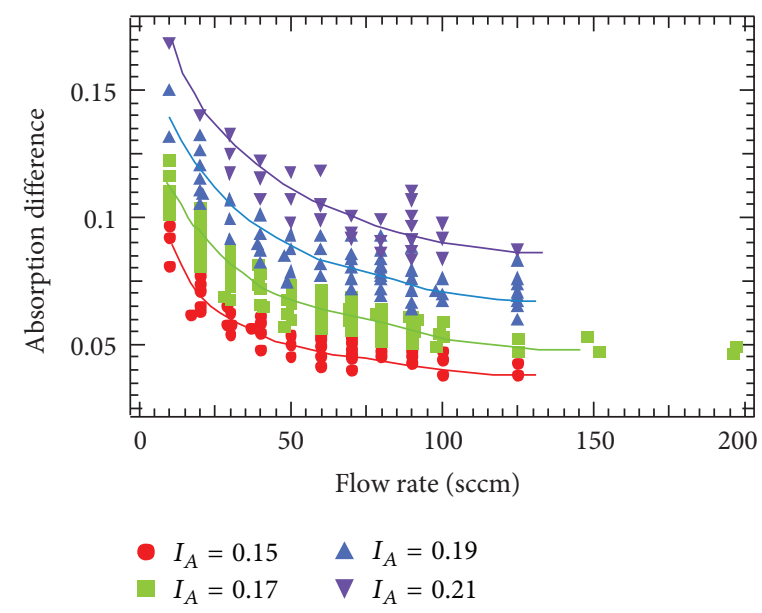

(c)

Figure 4: (a) The absorption of band-A, band-B, and band-C as a function of flow rate. (b) The difference in absorption between band-B and band-C as a function of flow rate. The absorption of band-A was kept at $0.17 \pm 0.01$. The insert shows the region at low flow rates. (c) The difference in absorption between band- $\mathrm{B}$ and band- $\mathrm{C}$ as a function of flow rate at several absorption strength of band-A. The variation of the band-A absorption was \pm 0.01 in all cases.

maintained at around 100 torrs, which lead to the absorption strength of band-A at around 0.17 .

The second and the third group of absorption lines are shown in Figure 3(b). These are due to the combination of $\sum_{u}^{+} \leftarrow \Sigma_{g}^{+}, \Pi_{g} \leftarrow \Pi_{u}, \Delta_{u} \leftarrow \Delta_{g}$, and $\Phi_{g} \leftarrow \Phi_{u}$ transitions [15]. To get a broad picture, we take the average strength of the absorption lines obtained from the low resolution spectra. The average strength within the range from 2284 to $2349 \mathrm{~cm}^{-1}$ is taken as the band-B absorption strength $I_{B}$ and that between 2349 and $2390 \mathrm{~cm}^{-1}$ as the band-C absorption strength $I_{C}$. Only the low resolution spectra are analyzed in the remaining part of the paper.

Figure 4(a) shows the absorption strength of band-A, band- $\mathrm{B}$, and band- $\mathrm{C}$ as a function of flow rate. The absorption strength of band-A was kept within the range of $I_{A}=0.17 \pm$ 0.01 throughout the flow rate changing from 0 to $2000 \mathrm{sccm}$. It is seen that relative to band-A, the absorption strength of band-B and band- $C$ first decreases when the flow rate increases from 0 to about $100 \mathrm{sccm}$. The decrease of strength then stops after the flow rate reaches $200 \mathrm{sccm}$ and beyond. In Figure 4(b), the difference in absorption strength between band-C and band-B, $I_{C}-I_{B}$, is plotted as a function of flow rate. Again, the strength of the band at higher frequencies decreases faster than the one at lower frequencies as the flow rate increases from 0 to about $100 \mathrm{sccm}$. The difference then remains nearly unchanged after the flow rate reaches beyond $100 \mathrm{sccm}$ and up to $2000 \mathrm{sccm}$. It is, therefore, clear that in the laminar flow regime, there is a continuous redistribution of population on different molecular energy levels as the flow rate increases. Such redistribution comes to an abrupt stop when the flow changes to turbulence. The population distribution then remains almost unchanged even up to the flow rate 10 times the laminar to turbulent transition.

Figure $4(\mathrm{c})$ shows $I_{C}-I_{B}$ as a function of flow rate for different constant band-A strength. $I_{C}-I_{B}$ is larger for larger $I_{A}$, that is, higher gas density, indicating that the population distribution of the gas depends on the intermolecular collisions. On the other hand, they all decrease at about the same 
rate with the increase of flow rate below $100 \mathrm{sccm}$ and level off at the flow rates beyond that critical point.

It is noted that in the flow rate versus differential pressure analysis, the laminar to turbulent transition occurs around $200 \mathrm{sccm}$, while in the absorption strength versus flow rate analysis, such transition seems to occur at $100 \mathrm{sccm}$. The main reason for the discrepancy is that the location where the infrared spectra were taken was close to the inlet of the cell, where there was a discontinuity of cross-section which could cause local unstable flow, while in the flow rate versus differential pressure analysis, it was the flow in the entire cell that was analyzed. The flow in the local region around the window could become turbulent at a lower flow rate $(100 \mathrm{sccm})$ before the flow in the entire cell became turbulent at higher flow rate $(200 \mathrm{sccm})$.

In conclusion, we have shown that the population of different excited states in carbon dioxide depends on its state of flow, that is, whether it is laminar or turbulent. Turbulence is associated with greater population of the excited states. This observation supports a quantum view of turbulence. Quantum turbulence, as it is sometimes called, has been observed in low-temperature physics [20-22]. Our results now show that even at room temperature, quantum concepts are important. In a future paper [23], it will be shown using already published data [9] that the rotational states of nitrogen render microflow in a Hinkle apparatus quantized. It now appears that turbulence itself may be quantum in nature [24].

\section{Acknowledgment}

This work was supported in part by the ERDT collaboration between the University of the Philippines and Columbia University (Amador Muriel).

\section{References}

[1] U. Frisch, Turbulence: The Legacy of A. N. Kolmogorov, Cambridge University, Cambridge, UK, 1995.

[2] O. A. Nerushev and S. A. Novopashin, "Rotation of molecules and the transition to turbulence," Journal of Experimental and Theoretical Physics Letters, vol. 64, no. 1, pp. 47-50, 1996.

[3] O. A. Nerushev and S. A. Novopashin, "Rotational relaxation and transition to turbulence," Physics Letters A, vol. 232, no. 34, pp. 243-245, 1997.

[4] S. A. Novopashin and A. Muriel, "Is the critical Reynolds number universal?" Journal of Experimental and Theoretical Physics Letters, vol. 122, no. 2, pp. 306-309, 1998.

[5] S. A. Novopashin and A. Muriel, "Molecule dependent turbulent memory," Physics Letters A, vol. 335, pp. 434-438, 2005.

[6] C. M. White and K. R. Sreenivasan, "Does molecular rotation affect the transition Reynolds number?" Physics Letters A, vol. 238, no. 6, pp. 323-327, 1998.

[7] C. J. Swanson, B. Julian, G. G. Ihas, and R. J. Donnely, "Pipe flow measurements over a wide range of Reynolds numbers using liquid helium and various gases," Journal of Fluid Mechanics, vol. 461, pp. 51-60, 2002.

[8] A. Muriel, "A molecular basis for the onset of turbulence," Journal of Vacuum Science \& Technology A, vol. 27, no. 2, article 315, 6 pages, 2009.
[9] L. D. Hinkle and A. Muriel, "Apparatus for laminar-turbulent transition in gases," Journal of Vacuum Science \& Technology A, vol. 23, no. 4, article 676, 5 pages, 2005.

[10] L. D. Hinkle, S. A. Novopashin, and A. Muriel, "Pressure dependence of laminar-turbulent transition in gases," Journal of Vacuum Science \& Technology A, vol. 24, no. 4, Article ID 068604JVA, pp. 1578-1583, 2006.

[11] A. Muriel, "Three related proposals for a theoretical definition of turbulence," Physica A, vol. 388, no. 4, pp. 311-317, 2009.

[12] M. P. Solon, P. Esguerra, and A. Muriel, "Turbulence in a gas laser," Physica A, vol. 388, no. 20, pp. 4361-4363, 2009.

[13] A. Muriel, "A molecular basis for the onset of turbulence," Journal of Vacuum Science \& Technology A, vol. 27, no. 2, article 315, 6 pages, 2009.

[14] L. S. Rothman and L. D. G. Young, "Infrared energy levels and intensities of carbon dioxide-II," Journal of Quantitative Spectroscopy and Radiative Transfer, vol. 25, pp. 505-524, 1981.

[15] A. Baldaci, V. M. Devi, D. -W. Chen, and K. N. Rao, "Absorption spectrum of carbon dioxide at $4.3 \mu \mathrm{m}$," Journal of Molecular Spectroscopy, vol. 70, pp. 143-159, 1978.

[16] V. Malathi Devi, D. C. Benner, C. P. Rinsland, and M. A. H. Smith, "Absolute rovibrational intensities of ${ }^{12} \mathrm{C}^{16} 16_{2}$ absorption bands in the 3090-3850 $\mathrm{cm}^{-1}$ spectral region," Journal of Quantitative Spectroscopy and Radiative Transfer, vol. 60, pp. 741-770, 1998.

[17] L. S. Rothman, "Infrared energy levels and intensities of carbon dioxide. Part 3," Applied Optics, vol. 25, no. 11, pp. 1795-1816, 1986.

[18] D. C. Benner, V. M. Devi, C. P. Rinsland, and P. S. FerryLeeper, "Absolute intensities of $\mathrm{CO}_{2}$ lines in the 3140-3410- $\mathrm{cm}^{-1}$ spectral region," Applied Optics, vol. 27, pp. 1588-1597, 1988.

[19] L. A. Gross and P. R. Griffiths, "Temperature estimation of carbon dioxide by infrared absorption spectrometry at medium resolution," Journal of Quantitative Spectroscopy and Radiative Transfer, vol. 39, no. 2, pp. 131-138, 1988.

[20] R. J. Donnely, Physica B, vol. 1, pp. 329-333, 2003.

[21] W. F. Vinen and J. J. Niemela, "Quantum turbulence," Journal of Low Temperature Physics, vol. 128, no. 5-6, pp. 167-231, 2002.

[22] R. J. Donnely and C. J. Swanson, “Quantum turbulence," Journal of Fluid Mechanics, vol. 173, pp. 387-429, 1986.

[23] C. L. Laguno and A. Muriel, Journal of Vacuum Science \& Technology A. In press.

[24] H. S. Aly, "Towards a better understanding of turbulence," CFD Letters, vol. 1, no. 1, pp. 2-3, 2009. 

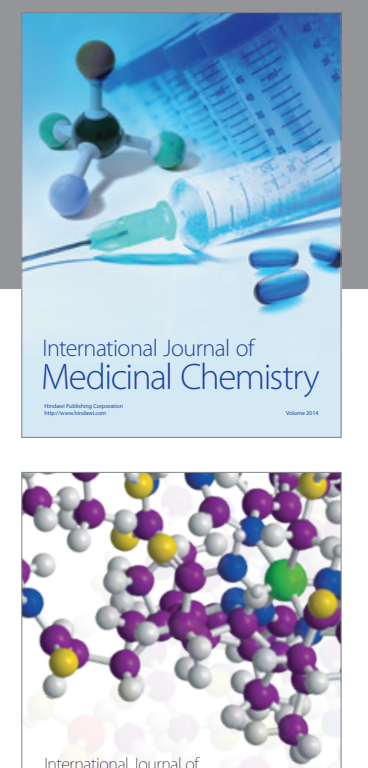

\section{Carbohydrate} Chemistry

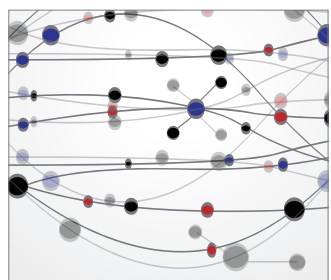

The Scientific World Journal
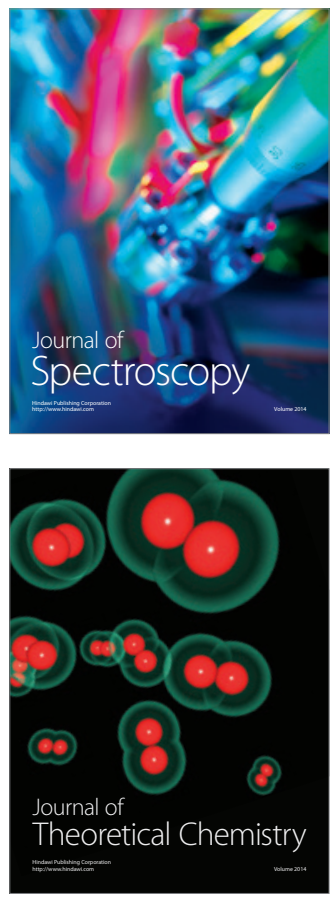
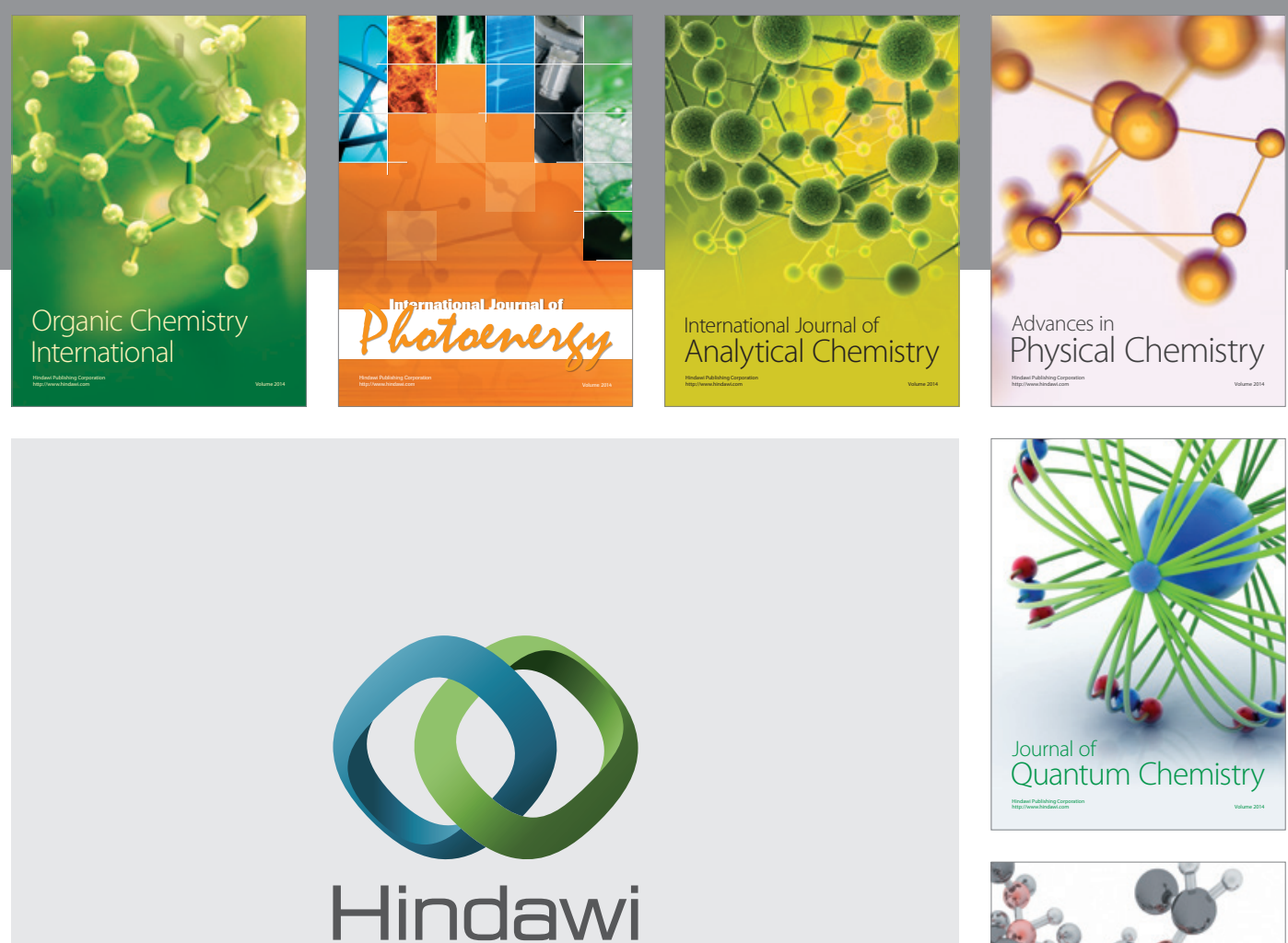

Submit your manuscripts at

http://www.hindawi.com

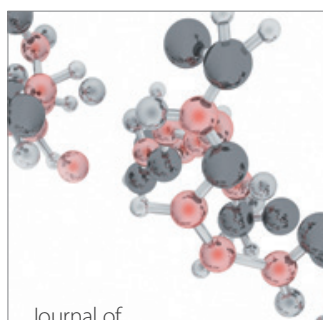

Analytical Methods

in Chemistry

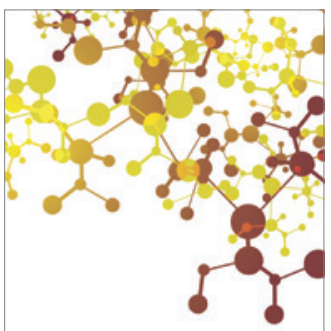

Journal of

Applied Chemistry

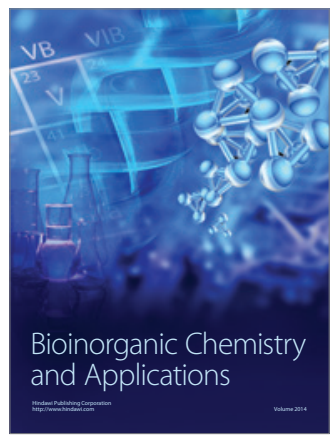

Inorganic Chemistry
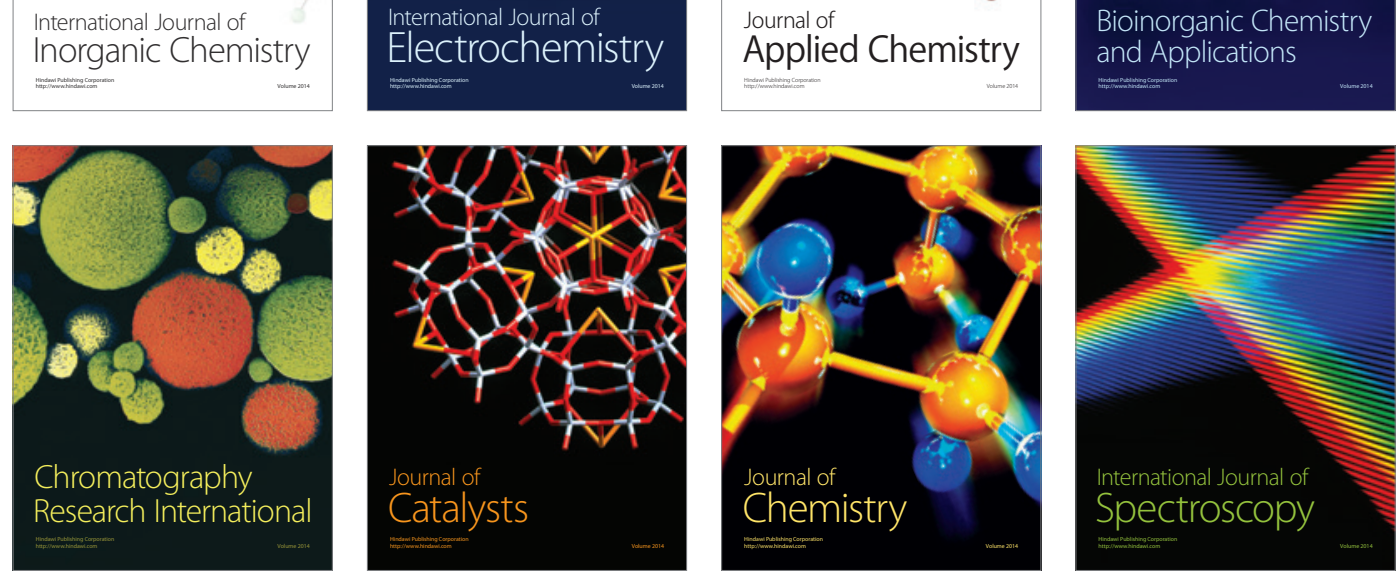